\title{
One of the Rare Causes of Acute Abdomen Leading to Subileus: Jejunal Diverticulitis
}

\author{
Elçin Aydın ${ }^{1}$, Hasan Yerli ${ }^{1}$, Tevfik Avcı², Tuğbahan Y1lmaz ${ }^{2}$, Hüseyin Gülay ${ }^{2}$ \\ ${ }^{1}$ Department of Radiology, Başkent University Zübeyde Hanım Hospital, İzmir, Turkey \\ ${ }^{2}$ Department of General Surgery, Başkent University Zübeyde Hanım Hospital, İzmir, Turkey
}

Background: Jejunal diverticulitis is one of the rare causes of acute abdomen generally seen in the elderly. Jejunal diverticulosis was defined as the herniation of the mucosa and the submucosa from the inside of the muscular layer of the bowel wall on the mesenteric side of the intestine.

Case Report: We presented the intraoperative and pathological findings of a 69 -year-old male patient who had presented with complaints about abdominal pain, nausea, and vomiting and been operated upon due to subileus and peritonitis induced by large-sized jejunal diverticulitis, along with his computed tomography (CT) findings.

Conclusion: Jejunal diverticulitis is uncommon and may be a disease which might be difficult to diagnose when it develops on the basis of the large-sized diverticula resembling intestinal ansae. To the best of our knowledge, the computed tomography and intraoperative findings of a case in which partial resection is applied to the jejunum due to subileus have not been previously presented in the literature.

Keywords: CT, ileus, jejunal diverticulitis
The rate of finding small intestinal diverticula in autopsies ranges from $0.06 \%$ to $1.3 \%$. Therefore, jejunal diverticulitis is a rather uncommon disease and may rarely be one of the causes of acute abdomen in the elderly in particular $(1,2)$. No surgical operation is performed and a palliative medical treatment is applied as long as no complication develops in symptomatic patients with jejunal diverticulitis. Some uncommon complications of jejunal diverticulitis such as perforation and an abscess have life-threatening potential and require an early surgical operation $(1,2)$. In this report, we presented the intraoperative and pathological findings of a case in which partial resection of the jejunum was performed due to subileus and peritonitis induced by large-sized jejunal diverticulitis as well as his computed tomography (CT) findings.

\section{CASE PRESENTATION}

Diffuse abdominal sensitivity and rebound were observed in the physical examination of a 69-year-old male patient who had presented to the emergency service with complaints about abdominal pain, nausea, and vomiting. Leukocytosis $(15,000 / \mu \mathrm{L})$ and high C-reactive proteins $(17.09 \mathrm{mg} / \mathrm{dL})$ were detected in his laboratory findings. We obtained upright abdominal X-ray before computed tomography (CT) and saw air-fluid levels and multiple dilated loops of the small bowel, which suggested ileus. The case was evaluated by means of oral and intravenous contrast-enhanced spiral abdominal CT. The examination was performed using a spiral CT (Somatom Balance; Siemens, Erlangen, Germany). Multiple diverticula were observed in the mesenteric segment in the small intestinal section at the jejunal level, thickening was observed in the diverticulum and on the neighboring bowel walls and linear densities were observed in the mesentery in the CT examination (Figure 1a, b). In the laparotomy, multiple diverticula were seen on the mesenteric side in the $100 \mathrm{~cm}$ jejunal segment starting from a point $20 \mathrm{~cm}$ distal to the ligament of Treitz (Figure 1c). The sizes of the diverticula ranged from 2 to $5 \mathrm{~cm}$. It was seen that two distinct diverticula were inflamed and that the omentum adhered to these regions. Widespread

This study was presented at the $35^{\text {th }}$ National Radiology Congress, 11-16 November 2014 Antalya, Turkey.

Address for Correspondence: Dr. Elçin Aydın, Department of Radiology, Başkent University Zübeyde Hanım Hospital, İzmir, Turkey

Phone: +905326419164 e-mail: elcinaydin09@yahoo.com.tr

Received: 18 March $2015 \quad$ Accepted: 19 June 2015 • DOI: 10.5152/balkanmedj.2016.141244

Available at www.balkanmedicaljournal.org 

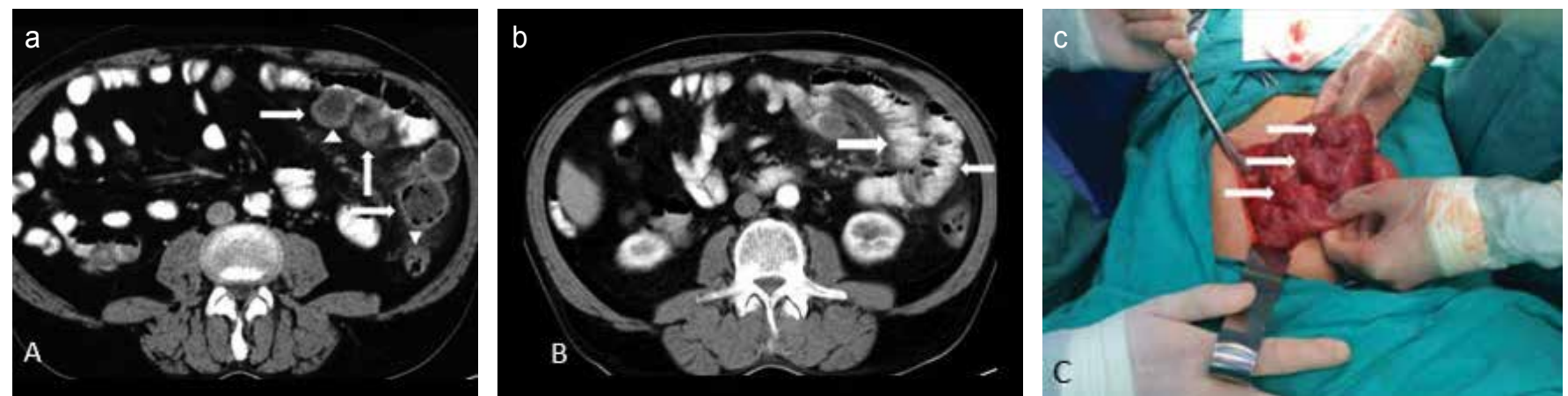

FIG. 1. a-c. Contrast-enhanced computed tomography images show multiple diverticula (a) (arrows), thickening in the diverticulum walls (arrowheads), and inflammation in the mesentery at the jejunal level (b). Mild dilatation was observed in the proximal small intestinal segments (arrows). In the laparotomy, multiple diverticula (arrows) were seen on the mesenteric side in the jejunal segment (c).

inflammation was observed in the segments where the diverticula were found, and mild dilatation was observed in the proximal small intestinal segments. The dense inflammation in the large-sized diverticula led to adhesions in the lumen, thereby narrowing the lumen. Thus, and in order to prevent the potential ileus from occurring in the future, resection was applied to this segment. In the pathological examination, a large number of diverticula leading to the intestinal lumen with diameters varying between 2 and $5 \mathrm{~cm}$ were detected along the mesenteric side. The mucosa appeared eroded and hemorrhagic on the interior surfaces of the diverticula, and he was diagnosed with diverticulosis/diverticulitis and peritonitis. Informed consent was obtained from the patient for the release of relevant information.

\section{DISCUSSION}

Jejunal diverticulosis was first defined as herniation of the mucosa and the submucosa from the inside of the muscular layer of the bowel wall on the mesenteric side of the intestine in 1794 by Sommering and later in 1807 by Sir Asley Cooper $(1,3)$. Jejunal diverticula are detected in autopsy series at rates ranging from $1.3 \%$ to $4.6 \%$, whereas the rate of radiological detection is between $0.02 \%$ and $2.3 \%(4,5)$. It is the proximal jejunum where a diverticulum is the most prevalent in the small intestine, and the incidence of diverticula decreases as one progresses towards the ileum. In addition, even though the diverticula with jejunal and ileal localization are seen 3 times less frequently than those found in the duodenum, they lead to complications 4 times more often $(1,6)$. When a diverticulum is detected in the jejunum, the rate of simultaneous presence of a diverticulum in another location is the most frequent in the colon and then in the duodenum, esophagus, stomach, and bladder at an descending frequency $(1,7)$. In our patient, diverticula were observed in the transverse colon during postopera- tive colonoscopy. Abnormal peristalsis, intestinal dyskinesia and high segmental intraluminal pressure are considered the causes of intestinal diverticulum. This pulsion-type pseudodiverticulum is seen on the mesenteric side of the small intestine, where the blood vessels perforate the muscular layer of the bowel wall and create weak areas $(4,8)$. The sizes of jejunal diverticula are generally reported to be several millimeters.

Diverticula are generally asymptomatic. Complaints of epigastric pain, abdominal sensitivity and gas occurring after eating have been described as the triad of 'gaseous dyspepsia' (1). Chronic abdominal pain, malabsorption, hemorrhage, diverticulitis and, rarely, obstruction and perforation can be seen as complications of jejunoileal diverticula $(1,8)$. No perforation was detected in our case. The sizes of diverticula ranged from 2 to $5 \mathrm{~cm}$ in our case, and the dense inflammation led to adhesions in the lumen and partially narrowed the lumen. In the post-resection pathological evaluation, the mucosa appeared eroded and hemorrhagic on the interior surface of the diverticula.

Detection of the presence of free air by means of direct radiography is an important finding in the diagnosis of perforation. Thickening of the bowel wall and the presence of intra-abdominal fluid can be revealed by means of ultrasonography. However, CT is the most important method of imaging to both differentiate diverticulitis from the other causes of acute abdomen and detect complications of diverticulitis. It is unknown whether an oral contrast medium is necessary during CT examination. Therefore, oral contrast-enhanced and nonenhanced series should be compared in future studies. This comparison appears difficult as jejunal diverticulitis is an uncommon disease. In our patient, we preferred making an examination with an oral contrast medium in order to achieve intestinal distension. Jejunal diverticulitis is seen as an inflamed diverticulum localized in the jejunum in CT and may or may not be accompanied by abscess formation or perforation. In the presence of perforation, CT is the most effective method for detecting intraperitoneal gas (9). 
Pathologies such as Crohn's disease, perforated neoplasm, small intestinal ulceration induced by non-steroid anti-inflammatory drugs, small intestinal perforation due to a foreign body, and traumatic intestinal hematoma should be considered with precedence in the differential diagnosis of jejunal diverticulitis (9). A conservative medical treatment can be performed in cases with uncomplicated jejunal diverticulitis, while the treatment is surgical in the presence of perforation or an abscess (9). Although no perforation or abscess was present in our case, widespread inflammation in the large-sized diverticula led to adhesions in the lumen and narrowed the lumen. Therefore, and in order to prevent potential cases of ileus from occurring in the future, resection was applied.

In conclusion, jejunal diverticulitis is uncommon and may be a disease which could be difficult to diagnose when it develops on the basis of the large-sized diverticula resembling intestinal ansae. Diverticula with an inflamed appearance detected at the jejunal level, wall thickening in the neighboring intestinal segments and lines and increases in density at the mesenteric fat levels during a CT examination should encourage clinicians to consider jejunal diverticulitis. Our case is the first in the literature in which partial resection was applied to the jejunum due to the subileus and peritonitis that developed on the basis of the large-sized jejunal diverticulitis.

Ethics Committee Approval: Ethics committee approval was received for this study from the The Institutional Ethics Committee.

Informed Consent: Written informed consent was obtained from patient who participated in this case.

Peer-review: Externally peer-reviewed.

Author contributions: Concept - E.A., H.Y., T.A.; Design - E.A., H.Y., T.Y.; Supervision - E.A., H.Y., H.G.; Resource - E.A., H.Y., H.G.; Materials - E.A., T.A., T.Y.; Data Collection and/or Processing
- E.A., H.Y., T.A.; Analysis and/or Interpretation - E.A., H.Y., T.A.; Literature Search - E.A., H.Y., T.Y.; Writing - E.A., H.Y., T.A.; Critical Reviews - H.Y., E.A., T.A.

Conflict of Interest: No conflict of interest was declared by the authors.

Financial Disclosure: The authors declared that this study has received no financial support.

\section{REFERENCES}

1. Singal R, Gupta S, Airon A. Giant and multiple jejunal diverticula presenting as peritonitis a significant challenging disorder. J Med Life 2012;5:308-10.

2. Woubet TK, Josef F, Jens H, Bartels M, Hauss J. Complicated small-bowel diverticulosis: A case report and review of the literature. World J Gastroenterol 2007;13:2240-2. [CrossRef]

3. Makris K, Tsiotos GG, Stafyla V, Sakorafas GH. Small intestinal nonmeckelian diverticulosis: clinical review. J Clin Gastroenterol 2009;43:201-7. [CrossRef]

4. Alam S, Rana A, Pervez R. Jejunal diverticulitis: imaging to management. Ann Saudi Med 2014;34:87-90.

5. Ross CB, Richards WO, Sharp KW, Bertram PD, Schaper PW. Diverticular disease of the jejunum and its complications. $\mathrm{Am}$ Surg 1990;56:319-24.

6. Wojciech S, Michel C, Stefania P, Demartines N. Acute ulcerative jejunal diverticulitis: Case report of an uncommon entity. World J Gastroenterol 2008;14:6265-7. [CrossRef]

7. Singh S, Sandhu HP, Aggarwal V. Perforated jejunal diverticulum: A rare complication. Saudi J Gastroenterol 2011;17:367. [CrossRef]

8. Akhrass R, Yaffe MB, Fischer C, Ponsky J, Shuck JM. Smallbowel diverticulosis: perceptions and reality. J Am Coll Surg 1997;184:383-8.

9. Macari M, Faust M, Liang H, Pachter HL. CT of jejunal diverticulitis: imaging findings, differential diagnosis, and clinical management. Clin Radiol 2007;62:737. [CrossRef] 